\title{
Prevalência de parasitas intestinais em habitantes do rio Negro, Estado do Amazonas, Brasil.
}

\author{
Luiz Cândido de Souza Dias ('); Júlio Dellome Filho $\left({ }^{2}\right)$; Marcilene Gomes Paes $\left({ }^{2}\right)$; Antônio Nogueira de \\ Farias $\left(^{2}\right)$ e João Carlos Sales Aguiar ${ }^{(2)}$.
}

\section{Resumo}

Os autores avaliaram a prevalência de enteroparasitas em habitantes de 4 localidades, às margens do rio Negro, no Estado do Amazonas. Utilizando-se os métodos de Lutz nas fezes de 213 indivíduos e de KatoKatz, em 154, as prevalências foram respectivamente, para ancilostomídeo $40,4 \%$ e $50,0 \%$, para $\mathbf{A}$. lumbricoides $54,5 \%$ e $65,6 \%$, para $\mathbf{T}$. trichiura $52,1 \%$ e $87,0 \%$ e para E. vermicularis $0,0 \%$ e $0,6 \%$. Em 190 amostras de fezes examinadas pela técnica de hematoxilina férrica, houve prevalências de $17,9 \%$ de E. histolytica, de $11,0 \%$ de E. coli, de $2,1 \%$ de $\mathbf{I}$. bütschlii, de $4,7 \%$ de E. nana e de $17,9 \%$ de G. lamblia. A prevalência de $\mathbf{S}$. stecoralis, em 147 indivíduos, foi de $1,4 \%$, pelo método de Baermann-Moraes. Em 161 amostras examinadas pelo método de Harada-Mori, constatou-se em 81 (50,3\%) presença de larvas, sendo $97,5 \%$ de $\mathrm{N}$. americanus. Pelo método de Kato-Katz, a intensidade de infecção por ancilostomídeos, em 71 indivíduos, foi leve $1<2599$ ovos/g de fezes) em 95,8\%; a carga parasitária por A. lumbricoides teve a mediana de $14160 \mathrm{ovos} / \mathrm{g}$, em 103 amostras; a mediana foi de 1320 ovos/g de fezes em 108 indivíduos portadores de T. trichiura. Os autores concluiram que a prevalência das parasitoses intestinais não diferem, grandemente, dos valores assinalados para região Amazônica e que foi leve a intensidade de parasitismo por vermes.

\section{INTRODUÇÃo}

Até hoje, não se conhece, com exatidão, a importância das parasitoses intestinais, na região Amazônica, quando, principalmente, se tenta avaliar a intensidade de parasitismo. Não há referência, na literatura, a inquéritos recentes, em larga escala, que possam definir o panorama. Entretanto, os dados apresentados por Vinha (1971), baseados no levantamento nacional de exames de fezes realizados pelo extinto DNERu (Departamento Naciona! de En- demias Rurais) em 1969, fornece-nos uma idéia da magnitude das parasitoses intestinais, nesta vasta área do território brasileiro. A prevalência de ancilostomídeos foi de $21,4 \%$, a de Ascaris lumbricoides de $88,3 \%$, a de Strongyloides stercoralis de $4,3 \%$, a de Enterobius vermiculares de $1,1 \%$, a de Trichuris trichiura de $62,0 \%$ e a de protozoários intestinais de $26,7 \%$. Estes dados baseiam-se em 67.182 exames de fezes.

Camillo-Coura (1970), Nohmi (1975, a, b) e Fraiha Neto (1977) relatam dados interessantes sobre parasitas intestinais na Amazônia, demonstrando que as prevalências são relati. vamente mais altas do que as registradas em outras regiões brasileiras.

O presente trabalho tentou avaliar a prevalência das parasitoses intestinais, qualitativa e quantitativamente, em amostragem de habitantes das margens do rio Negro, no Estado do Amazonas.

\section{MATERIAL E MÉTODOS}

A amostra estudada era composta de habitantes de quatro localidades (Novo Airão, Moura, Carvoeiro e Barcelos) situadas às margens do rio Negro, no Estado do Amazonas. Foram coletadas amostras de fezes, em fevereiro de 1980 , de 213 indivíduos (100 do sexo masculino e 113 do feminino), sendo que $61 \%$ apresentaram idade entre 1 mês e 15 anos.

Os parasitas intestinais foram identificados, utilizando-se as seguintes técnicas: a) método de sedimentação qualitativa de Lutz

(1) - Universidade Estadual de Campinas, Instituto de Biologia, Campinas (SP).

(2) - Universidade do Amazonas. Manaus. 
(1919); b) método quantitativo de Kato-Katz (Katz et al., 1972); c) método de Harada \& Mori (1951). A classificação das larvas foi baseada na morfologia de larvas filarióides de $3 .^{\circ}$ estágio (Corrêa et al., 1979); d) método de Baermann-Moraes (in Pessoa \& Martins, 1977); e) coloração por hematoxilina férrica (in Pessoa \& Martins, 1977).

Para os métodos (a) e (e), fazia-se uma lâmina por indivíduo para pesquisa de parasitos. A avaliação quantitativa das infecções por ancilostomídeos, $A$. lumbricoides e $T$. trichiura, pelo método de Kato-Katz, foi realizada em 3 lâminas por indivíduo.

\section{REsUltados}

Examinaram-se fezes de 147 indivíduos pelo método de Baermann-Moraes, detectandose $2(1,4 \%)$ com larvas de $S$. stercoralis.

Utilizando-se a técnica de Harada-Mori, encontraram-se, no exame de 161 pacientes, $81(50,3 \%)$ com larvas das seguintes espécies: $79(97,5 \%)$ com larvas de Necator americanus, $1(1,2 \%) \operatorname{com} N$. americanus e S. stercoralis e $1(1,2 \%)$ com N. americanus e Ancylostoma duodenale.

$\mathrm{Na}$ Tabela 1, encontram-se os resultados da prevalência dos enteroparasitas, segundo técnicas de Lutz, Kato-Katz e hematoxilina férrica.

A intensidade de infecção por ancilostomídeos, avaliada em 71 indivíduos, pelo método de Kato-Katz foi leve $(<2599$ ovos/g de fezes) em $95,8 \%$ e moderada (2 600 a 12599 ovos $/ g$ ) em $4,2 \%$, segundo classificação proposta por Pessoa \& Martins (1977).

Nas fezes de 103 pacientes, foi avaliada a quantidade de ovos de $A$. lumbricoides, pelo método de Kato-Katz, sendo o valor mediano de 14160 ovos por grama de fezes e a média aritmética de 27 309. Para 108 indivíduos portadores de $T$. trichiura, a mediana foi de 1320 e a média aritmética de 1972 .

Dada a insuficiência de material coletado, não foi possivel realizar todas as técnicas propostas na amostragem de 213 pacientes.

\section{DISCUSSÃO}

Apesar das diferentes técnicas de amostragem de exame de fezes utilizadas em inquéritos sobre enteroparasitas no Estado do Amazonas, nota-se que nossa percentagem de indivíduos portadores de ancilostomídeos oscilou entre 40,0 e $50,0 \%$ (Tabela 1), sendo superior à registrada por Vinha (1971), cuja taxa foi de $15,6 \%$. Segundo o mesmo autor, a percentagem nacional para esta helmintíase foi de $26,5 \%$. Já em relação ao $A$. lumbricoides nossos valores, de 54,0 a $65,0 \%$ (Tabeia 1), são inferiores aos dados de Vinha (1971) que assinalou $94,0 \%$ de infectados no Estado do Amazonas e $59,5 \%$ para o território brasileiro. A prevalência de $T$. trichiura, com valores de 52,0 a $87,0 \%$ (Tabela 1), aproxima-se do asnalado por Vinha (1971) de 59,9\% para o Estado do Amazonas. Para o território nacional, esta parasitose atinge cerca de $35,9 \%$ da população (Vinha, 1971).

Com referência aos demais helmintos, nossos valores equivalem aos do citado autor.

A baixa prevalência de $E$. vermiculares (Tabela 1) não reflete a realidade, dada a impropriedade dos métodos aqui utilizados.

A avaliação do parasitismo por S. stercoralis realizada por três técnicas (BaermannMoraes, Harada-Mori e Lutz) sempre demonstrou valores inferiores a $2,3 \%$, ligeiramente acima dos de Vinha (1971) para o Estado do Amazonas, que era de $0,7 \%$, utilizando-se técnicas laboratoriais não apropriadas. Parecenos que as condições ecológicas para manutenção do ciclo de $S$. stercoralis não seriam sa. tisfatórias na região amostrada.

Constatamos que, na população estudada entre os 81 indivíduos que apresentavam larvas nas fezes pelo método de Harada-Mori, todos possuiam exemplares de $N$. americanus. Nossos dados concordam com o fato largamente conhecido de que este menatóide predomina em todo o Brasil. A. duodenale, detectado em apenas um paciente, é encontrado em áreas onde habitam imigrantes europeus e asiáticos (Pessoa \& Martins, 1977; Asami et al., 1970; Marzochi \& Chieffi, 1978; Corrêa et al., 1979). 
TABELA 1 - Prevalência de parasitas intestinais em habitantes do rio Negro, Estado do Amazonas, avaliada pelos métodos de Lutz, Kato-Katz e hematoxilina férrica - fevereiro de 1980

\begin{tabular}{|c|c|c|c|c|c|c|c|c|c|c|c|c|c|c|c|c|c|c|c|c|c|c|c|}
\hline \multirow{3}{*}{ Métodos } & \multirow{3}{*}{$\begin{array}{l}\mathrm{N} . \\
\text { exami- } \\
\text { nados }\end{array}$} & \multicolumn{16}{|c|}{ PARASITAS INTESTINAIS } & & & & & & \\
\hline & & & $\begin{array}{l}\text { siti- } \\
\text { os }\end{array}$ & $\begin{array}{l}\text { An } \\
\text { tom }\end{array}$ & $\begin{array}{l}\text { cilos- } \\
\text { ídeos }\end{array}$ & $\begin{array}{r}\text { A. lu } \\
\text { coi }\end{array}$ & $\begin{array}{l}\text { mbri- } \\
\text { des }\end{array}$ & trich & i. & & & $\begin{array}{r}\text { E. v } \\
\text { cul }\end{array}$ & $\begin{array}{l}\text { rmi- } \\
\text { iris }\end{array}$ & $\begin{array}{l}\text { E. I } \\
\text { ly }\end{array}$ & $\begin{array}{l}\text { isto- } \\
\text { ica }\end{array}$ & \multicolumn{2}{|c|}{$\begin{array}{l}\text { E. } \\
\text { coli }\end{array}$} & \multicolumn{2}{|c|}{$\begin{array}{l}\text { I. büts- } \\
\text { chlii }\end{array}$} & \multicolumn{2}{|c|}{$\begin{array}{c}\text { E. } \\
\text { nana }\end{array}$} & \multicolumn{2}{|c|}{$\begin{array}{c}\text { G. } \\
\text { lamblia }\end{array}$} \\
\hline & & $\mathrm{N} .^{\circ}$ & $\%$ & N. & $\%$ & N.: & $\%$ & N. & $\%$ & N. & $\%$ & N. & $\%$ & $\mathrm{~N} \mathrm{0}^{\circ}$ & $\%$ & N. & $\%$ & N. & $\%$ & N. & $\%$ & N. & $\%$ \\
\hline Lutz & 213 & 191 & 89,7 & 86 & 40,4 & 116 & 54,5 & 111 & 52,1 & 5 & 2,3 & .. & .. & 31 & 14,5 & 27 & 12,7 & 3 & 1,4 & 10 & 4,7 & 19 & 8,9 \\
\hline Kato-Katz & 154 & 142 & 92,2 & 77 & 50,0 & 101 & 65,6 & 134 & 87,0 & .. & .. & 1 & 0,6 & .. & .. & .. & . & . & . & .. & . & .. & $\cdots$ \\
\hline $\begin{array}{l}\text { Hematoxilina } \\
\text { férrica }\end{array}$ & 190 & 105 & 55,3 & .. & .. & .. & .. & .. & .. & .. & $\cdots$ & $\ldots$ & .. & 34 & 17,9 & 21 & 11,0 & 4 & 2,1 & 9 & 4,7 & 34 & 17,9 \\
\hline
\end{tabular}


Em nossa área de estudo, a presença destes imigrantes atualmente é rara. Sabe-se que ao redor de 1666, foi iniciada na área, a colonização por portugueses (Roque, 1967 . Segundo Marzochi \& Chiefíi (1978), acredita-se que um contingente de imigrantes, após permanência por algum tempo, em certa região, começa a demonstrar padrão de infecção por ancilostomídeo, semelhante aos dos habitantes autóctones.

A intensidade de parasitismo por $N$. americanus avaliada em 71 indivíduos foi de grau leve em $95,8 \%$. Este dado nos faria supor que as condições edáficas não sejam suficientemente favoráveis a possibilitar intensidades mais elevadas de parasitismo, a despeito de as condições sanitárias, de temperatura e de umidade serem satisfatórias. Por outro lado, qualitativamente, a prevalência de ancilostomídeos foi alta, segundo técnicas de HaradaMori, Lutz e Kato-Katz (Tabela 1).

A medida da intensidade de infecção por A. lumbricoides e $T$. trichiura carece de estudos mais detalhados. Os resultados apresentados, na literatura, baseiam-se em valores expressos pela média aritmética que ao nosso ver, não representa a realidade, pois individualmente, os valores apresentam grande variação. Acreditamos que a mediana nos fornece, nestes casos, uma idéia mais precisa.

Com relação ao Ascaris, considerando o cálculo proposto por Pessoa \& Martins (1977) e o valor mediano de 14600 ovos por grama de fezes, estima-se em 14 o número de vermes por indivíduos em nossa amostragem. Esta cifra não nos parece alta, pois geralmente se encontram de 4 a 10 Ascaris no lume do intestino delgado por indivíduo, havendo casos extremos de 500 a 600 vermes (Pessoa \& Martins, 1977). Partindo-se do valor da média aritmética (27 309), nossa estimativa é inferior a de Pessoa \& Pascale (1938) que encontraram $34055 \mathrm{ovos} / \mathrm{g}$ de fezes em indivíduos residentes no litoral norte do Estado de São Paulo. Os mesmos autores relatam média aritmética de $6947 \mathrm{em}$ habitantes de fazendas no planalto paulista.
$\mathrm{O}$ número mediano de ovos de $T$. trichiura por grama de fezes foi de 1320 . Considerando em 200 o número médio de ovos postos por grama de fezes e por verme (Pessoa \& Martins, 1977), teríamos cerca de 7 fêmeas por indivíduo. Este númeró é considerado baixo para produzir sintomas clínicos (Maldonado, 1965).

Nossas estimativas das cargas $r$ drasitárias para $A$. lumbricoides e $T$. trichiv a devem ser vistas com reserva, pois parti los de suposições com limites amplos de rros. No entanto, estas avaliações nos ' رrnecem uma idéia das cargas parasitárias $m$ um grupo populacional. Acreditamos q' estes dados são importantes na epidemir gia das geohelmintíases complementandr ,s resultados qualitativos.

A prevalência $s$ protozoários intestinais será comentada acordo com os valores obtidos pela her uxilina férrica, método de coloração sabi iente mais específico.

Iniciah, nte, a positividade para protozoários intestinais de $55,3 \%$ (Tabela 1) corrobora com a tese de insalubridade do meio em que vive a população estudada, insalubridade, já detectada pelas outras técnicas de exame de fezes. A taxa de infecção por $E$. histolytica foi de $17,9 \%$ (Tabela 1). Segundo Pessoa \& Martins (1977), esta taxa varia de 27,0 a $4,0 \%$, no Estado do Amazonas, baseada em levantamentos efetuados em remota data (1922 e 1947). Mais recentemente, Nohmi (1975 a, b e c), no Território Federal do Amapá, registrou E. histolytica em 8 a $18,0 \%$ das fezes da população da cidade de Macapá.

Com relação aos demais protozoários intestinais, nossos dados são semelhantes aos relatados por outros autores no Brasil (Pessoa \& Martins, 1977).

Deixamos de comparar a especificidade e a sensibilidade das diferentes técnicas parasitológicas ora utilizadas, pois o material fornecido pelos pacientes muitas vezes não era suficiente para alcançar este objetivo. Por outro lado, cada técnica executada tem especificidade diferente para certo grupo de parasitas. 
Quanto à prevalência das parasitoses, de acordo com a faixa etária e sexo, não notamos diferenças significativas que justificassem aqui sua análise.

Os resultados permitem-nos concluir que a prevalência das parasitoses intestinais, na amostra populacional de habitantes do rio $\mathrm{Ne}$ gro, não difere grandemente dos valores assinalados para outra região Amazônica; a infecção por $S$. stercoralis foi pequena a despeito da observância de técnicas apropriadas; a intensidade de parasitismo por ancilostomídeos, $A$. lumbricoides e $T$. trichiura foi baixa; a espécie de ancilostomídeo predominante foi $N$. americanus.

\section{AGRADECIMENTIOS}

Os autores agradecem ao Prof. Dr. Heitor Dourado, diretor do Instituto de Medicina Tropical de Manaus, pelas facilidades oferecidas na coleta do material e à Sra. Raimunda Lenice da Silva pelo auxílio na realização dos exames de fezes.

\section{SUMMARY}

The authors evaluated the prevalence of intestinal parasites among inhabitants at four localities along the Rio Negro. Results for the Lutz method performed on fecal samples of 213 persons and Kato-Katz method, in 154 , gave prevalences of, respectively, $40.4 \%$ and $50.0 \%$ for hookworms, $54.5 \%$ and $65.6 \%$ for $\mathbf{A}$. lumbricoides, $52.1 \%$ and $87.0 \%$ for $\mathbf{T}$. trichiura and $0.0 \%$ and $0.6 \%$ for E. vermicularis. Results from iron-hematoxylin staining carried out with 190 samples gave prevalences of $17.9 \%$ for E. histolytica, $11.0 \%$ for E. coli, $2.1 \%$ for I. bütschlii, $4.7 \%$ for E. nana and 17.9 for G. Iamblia. Infection by $\mathbf{S}$. stercoralis, in 147 persons, was $1.4 \%$, using the Baermann-Moraes method. The Harada-Mori method used in 161 patients revealed $50.3 \%$ of those with larvae, of which $97.5 \%$ were N. americanus. The intensity of parasitism by hookworms, as evaluated by the Kato-Katz method in 71 patients, was mild $(<2,599$ eggs $/ \mathrm{g}$ of stool) in $95.8 \%$. In 103 samples with A. lumricoides the median was 14,160 eggs $/ \mathrm{g}$ and the median for $\mathrm{T}$. trichiura in 103 stools was $1,320 \mathrm{eggs} / \mathrm{g}$. The authors concluded that the prevalence of intestinal parasites did not diffed greatly from earlier data from the the Amazonian region, and the intensity of parasitism by worms was mild.

\section{REFERENCIAS BIBLIOGRÁFICAS}

ASAMI, K.; ENOMOTO, Y.; MIURA, S.

1970 - Infestações por ancilostomídeos e Strongyloides stercoralis em Pernambuco. Inquérito baseado na identificação das larvas. Revista do Instituto de Medicina Tropical dr São Paulo, 12: 31-35.

CAMILO-COURA, Léa

1970 - Contribuição ao Estudo das geohelr antíases. Tese de Concurso à Livre Docê jia, Faculdade de Medicina, Universida', Federal do Rio de Janeiro, Ed. do Al r, Rio de Janeiro, $215 p$.

CORREA, L.L.; SILVA, M.I.P.G.; \& /A, R.M.;

DIAS, R.M.D.S,

1979 - Ancylostoma duo ale e Necator americanus: diagnóstir siferencial das larvas infestantes e valência em amostras fecais prove' des da Grande São Paulo. Revista do atuto Adolfo Lutz, 39: 145-153.

FRAIHA NETO, $\vdash$

1977 - $\mathrm{Pa}$ : лa atual das parasitoses na Amazônia. Belém, SUDAM, 36p. ilust.

HARADA, Y. \& MORI, O.

1951 - A simple culture method of Ancylostoma duodenale. Medical Biology, 20: 65-67.

KATZ, N.; CHAVES, A.; PELLEGRINO, J.

1972 - A simple device for quantitative stool thicksmear technique in Schistosomiasis mansoni. Revista do Instituto de Medicina Tropical de São Paulo, 14: 397-400.

LUTZ, A.

1919 - O Schistosoma mansoni e a Schistosomose segundo observações feitas no Brasil. Memórias do Instituto Oswaldo Cruz, 11: 121144.

MALDONADO, J.F.

1965 - Helmintíasis del hombre in America. Editorial Científivo-Medica Barcelona (España).

MARZOCHI, M.C.A. \& CHIEFFI, P.P.

1978 - Estudos dos fatores envolvidos na disseminação dos enteroparasitas. IV - Distribuição do Necator americanos e do Ancylostoma duodenale na população urbana e rural do município de Londrina, Paraná, Brasil. Revista do Instituto de Medicina Tropical de São Paulo, 20:36-40.

NOHMI, N.

1975 - Parasitoses intestinais; contribuição ao seu estudo em Macapá, Território Federal do Amapá. Boletim do Hospital-Escola São Camilo e São Luiz, 8: 13-18. 
1975a - Parasitoses intestinais; inquérito coprológico em dois grupos de população de Macapá. Boletim do Hospital-Escola São Camilo e Săo Luiz, 8: 19-39.

1975b- Parasitoses intestinais; levantamento segundo grupos etários, no período de dezembro de 1968 a dezembro de 1972 em Macapá, Território Federal do Amapá. Boletim do Hospital-Escola São Camilo e São Luiz, 8: $49-59$.

PESSÔA, S.B. \& MARTINS, A.V.

1938 - Sobre a intensidade e prevalência do Ascaris lumbricoides e Trichuris trichiura, em algumas zonas do Estado de São Paulo. São Paulo Médico, 1: 93-201.
PESSÔA, S.B. \& PASCALE, $H$.

1977 - Pessôa Parasitologia Médica - 10. ed. Pio de Janeiro. Ed. Guanabara Koogan S.A.

ROQUE, C.

1967 - Grande Enciclopédica da Amazônia Belém, Amazônia Editora Ltda. 6 v.

VINHA, C.

1971 - Incidência, no Brasil, de helmintos transmitidos pelo solo. Rotina coproscópica do exDepartamento Nacional de Endemias Rurais. Revista Brasileira de Malariologia e Doenças Tropicais, 23: 3-18.

(Aceito para publicação em $02 / 12 / 81$ ) 